\title{
Internet of Things Management Mode and Multi-dimensionally Collaborative Evaluation Method for Agricultural Product Logistics Service
}

\author{
Gao Jie $^{1}$ and Zheng Mingliang ${ }^{1}$ \\ ${ }^{1}$ School of Economics and Management, Weifang University, \\ Weifang Shandong, 261061, China \\ gaojierkeke@163.com
}

\begin{abstract}
It aims at group incentive and alliance scale of collaborative logistics allance. It defines the connotation of collaborative logistics alliance based on theorles related to domestic and overseas logistics alliance and it establishes group incentive model under single alliance leader and multiple alliance leaders basea ondouble layer principal-agent theory. Solution shows that, in group incentive moded of logistics alliance, incentive coefficient under one alliance leader enterprise is the same as that under alliance leader decision-making system containing several enterp ises. Group scale of logistics alliance can be determined when certainty equivalent wealth of alliance teader decision-making system and alliance member enterprise is equal to their respective retained income.
\end{abstract}

Keywords: Collaborative logistics CInternet of Things; Scale design; Agricultural product; Multi-dimensional evaluatiôn

\section{Introduction}

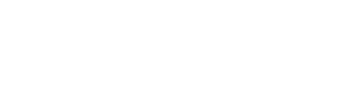

Development of modemagriculturalleproduct logistics requires logistics integration and logistics alliance is an important way to realize integrated agricultural product logistics and also an important channel to reduce logistics cost and to reinforce cooperation among logistics enterprises. At present, researches of most domestic scholars on logistics alliance mainly focus on the connotation, formation mechanism, cooperative relationship, partner selection, benefit distribution and knowledge transfer etc. It is mentioned in the literature that logistics alliance is a loose group constituted by several independent logistics enterprises to complete specific logistics tasks and there is no control or affiliation relationship among alliance partners and it is a form of organization between logistics enterprise and market transaction relationship. Current researches does not study group incentive problem of collaborative logistics alliance based on double layer principal-agent theory. Therefore, the thesis will introduce group incentive model into logistics alliance and wil establish group incentive model of collaborative logistics alliance based on couble-layer principal-agent theory to solve "hitchhike" problem in group management. It analyzes monitoring cost of alliance leader enterprise (body) in monitoring process of "hitchhike" and discusses scale design problem of logistics alliance based on this. Therefore, the thesis studies a significant topic having theoretical exploration and practical guidance.

\section{Connotation Definition of Collaborative Logistics Alliance}

As complexity, integrality and high risk of modern agricultural product logistics service outsourcing increase, more and more logistics enterprises establish various logistics alliances to reduce transaction cost, to reduce uncertainty, to complement each 
other's advantages and to generate collaborative effect. Xu Yang etc. [2] think logistics alliance is logistics enterprise strategy alliance with logistics as basis for cooperation. It refers to the process that, in order to realize target of logistics strategy, two logistics enterprises or above form an enterprise combination to complement each other's advantages, to trust each other, to undertake risks and share interests jointly through various agreements and contracts. Wang Ruogang etc. [8] think virtual logistics alliance is the process that two logistics enterprises or above keep independence and form a intensification logistics partnership which is stable temporarily through equity participation or contract combination so as to expand logistics market, reduce logistics cost and increase logistics efficiency together. Virtual logistics enterprise alliance is a kind of dynamic alliance. Dominant enterprise establishes logistics enterprise alliance according to specific logistics business requirement and the alliance will collapse after the task is completed. In the various logistics alliances, collaborative logistics alliance is a common form. In this kind of logistics alliance, one alliance leader enterprise or "alliance" leader decision-making system" formed by several logistics enterprises formulates rues of the game and other member enterprises decide whether to participate in operation of logistics service. In order to complete one logistics task, combination of various logistics capabilities may need to be provided and logistics enterprise must conbines internal capacity and external capacity of the enterprise in proper way to meet customer need and ensure enterprise profit. Mahoney and Pandian differentiate "competitive collaboration" and "particular collaboration" and proves that organization can ceate value when it combines different capacities closely and this kind of particular collaboration promotes the need for combination. Based on above analysis and definition of related logistics alliances, the thesis defines collaborative logistics alliance as follows:

(1) Alliance leader of collaborative logis ics alliance may be an enterprise or "alliance leader decision-making system" constituted by several powerful logistics enterprises;

(2) Collaborative logistics alliance is a kind of alliance form for division and cooperation among different logistics enterprises;

(3) In collaborative logistics alliance, the alliance leader combines logistics capabilities of different logistics enterprises to create value;

(4) Collaborative logisties alliance is a kind of interim organization form between logistics enterprise and markeg transaction;

(5) Collaborative logistics alliance meets essential features of general strategic alliance: in order to reach the common strategic target, several logistics enterprises form a loose and networked alliance having complementary advantages, coordination and distribution of responsibilities and joint action. They cooperate with each other and undertake risks and share interests jointly based on joint action.

\section{Construction and Solution of Group Incentive Model}

\subsection{Fundamental Assumption and Parameter Setting}

The thesis provides the following fundamental assumptions according to connotation definition of agricultural product logistics alliance: (1) assume there is 1 alliance leader enterprise and $n$ member enterprises in the collaborative logistics alliance and logistics task is completed by all member enterprises; (2) principal agent relationship between customer enterprise and alliance leader enterprise and member enterprises are as follows: customer enterprise relegates logistics task to alliance leader enterprise with customer enterprise as the first principal, alliance leader enterprise as the second principal and member enterprises as the agents. The thesis makes the following assumptions based on above assumptions.

Assumption 1: according to assumption 2, assume that effort level of member enterprise in logistics alliance on logistics service is $e_{i}$, the first principal customer 
enterprise and alliance leader enterprise can not observe effort level of member enterprise directly.

Assumption 2: effort level selection of each member enterprise on logistics task is oneoff and the effort $\operatorname{cost} C\left(e_{i}\right)=\frac{1}{2} b e_{i}^{2}$, where $\mathrm{b}$ is parameter for effort cost of member enterprise and it is a constant.

Assumption 3: assume $R\left(e_{i}\right)=\lambda_{i} e_{i}$ is the effort output of the $i$ th member enterprise and $\lambda_{i}$ is the effort output coefficient of the $i$ th member enterprise. Output of whole logistics alliance at this time is $R=\sum_{i=1}^{n}\left[R\left(e_{i}\right)+\varepsilon_{i}\right]$, where $\varepsilon_{i}$ is the output random variable of the $i$ th member enterprise and it obeys normal distribution with variance of $\sigma_{i}^{2}$.

Assumption 4: set $\alpha$ as constant return and $\beta_{1}$ as income distribution coefficient set by customer enterprise, $\beta_{2}$ as income distribution coefficient set by alliance leader enterprise, $c$ as monitoring cost of alliance leader enterprise for logistics service of member enterprise and $\bar{\alpha}_{0}$ as retained income of member enterprise. As ume constant return of alliance leader enterprise is equal to that of member enterprise and the constant return is shared by member enterprises.

Assumption 5: assume customer enterprise and alliance leaden enterprise are risk neutral and alliance member enterprises are risk aversion. Utility function of member enterprise is $u(x)=-\exp \left(-\rho_{i} x_{i}\right)$, where $x_{i}$ is actual income of the $i$ thember enterprise and $\rho_{i}$ is the risk aversion of the $i$ th member enterprise. Its expression is $\rho=-\frac{u^{\prime \prime}\left(x_{i}\right)}{u^{\prime}\left(x_{i}\right)} ; x_{i} \sim N\left(m_{2}, v^{2}\right)$. Risk cost of alliance memberenterprise is $\frac{1}{2} \rho_{i} \beta_{1}^{2} \beta_{2}^{2} \sigma_{i}^{2}$.

\subsection{Construction and Solution of Group Incentive Model}

On the basis of the assumptron and paraneter setting above, construct the following group incentive model (1)according to principal agent theory.

$$
\begin{aligned}
& \max _{\alpha, \beta_{1}}\left(1-\beta_{1}\right)\left(R-\sum_{i=1}^{n+1} \alpha\right) \\
& \text { s.t. } \max _{\alpha, \beta_{1}, \beta_{2}}\left[\alpha+\beta_{1}\left(1-\beta_{2}\right)\left(R-\sum_{i=1}^{n+1} \alpha\right)-c\right] \\
& \text { s.t.(IR) } \alpha+\beta_{1} \beta_{2}\left(R(e)-\frac{\alpha}{2} \rho_{i} \beta_{1}^{2} \beta_{2}^{2} \sigma_{i}^{2}-\frac{1}{2} b e_{i}^{2} \geq \bar{\alpha}_{o}\right. \\
& \text { (IC) } \max \left[\alpha+\beta_{1} \beta_{2}\left(R\left(e_{i}^{*}\right)-\frac{\alpha}{n}-\alpha\right)-\frac{1}{2} \rho_{i} \beta_{1}^{2} \beta_{2}^{2} \sigma_{i}^{2}-\frac{1}{2} b e_{i}^{*}\right] \quad(i=1,2, \cdots, n)
\end{aligned}
$$

(IR) and (IC) are incentive compatible constraint and participation constraint respectively. (1) First formula represents expected revenue of client enterprises. Second formula represents expected revenue of alliance leader enterprise.

According to participation constraint in model (1), it can be calculated that:

$\beta_{1}=\frac{b e_{i}}{\beta_{2} \lambda_{i}}, \quad \beta_{2}=\frac{b e_{i}}{\beta_{1} \lambda_{i}}$

Derive $e_{i}$ from $\beta_{1}, \beta_{2}$ to calculate:

$\frac{\partial \beta_{1}}{\partial e_{i}}=\frac{b}{\beta_{2} \lambda_{i}}, \frac{\partial \beta_{2}}{\partial e_{i}}=\frac{b}{\beta_{1} \lambda_{i}}$

Deterministic equivalent income of logistics alliance is:

$$
\sum_{i=1}^{n+1} \alpha+\beta_{1}\left(\sum_{i=1}^{n} \lambda_{i} e_{i}-\sum_{i=1}^{n+1} \alpha\right)-\frac{1}{2} \beta_{1}^{2} \beta_{2}^{2} \sum_{i=1}^{n} \rho_{i} \sigma_{i}^{2}-\frac{1}{2} b \sum_{i=1}^{n} e_{i}^{2}-c
$$

Use formula (1) to derive $e_{i}$ from formula (4) and command it to be zero to calculate: 
$\beta_{2}^{*}=\frac{\lambda_{i}^{2}}{\lambda_{i}^{2}+b \sum_{i=1}^{n} \rho_{i} \sigma_{i}^{2}}$

Sum of deterministic equivalent incomes of client enterprises and logistics alliance is:

$\sum_{i=1}^{n} \lambda_{i} e_{i}-\frac{1}{2}\left(\sum_{i=1}^{n} \rho_{i} \sigma_{i}^{2}\right) \beta_{2} \beta_{1}^{2}-\frac{1}{2} b \sum_{i=1}^{n} e_{i}^{2}-c$

Use formula (6) to derive $e_{i}$ and command it to be 0 by combining formula (2) to calculate:

$\lambda_{i}-\frac{2 b}{\lambda_{i}}\left(\sum_{i=1}^{n} \rho_{i} \sigma_{i}^{2}\right) \beta_{1} \beta_{2}-\beta_{1} \beta_{2} \lambda_{i}=0$

It can be calculated from formula (7) that:

$$
\beta_{1}^{*}=\frac{\lambda_{i}^{2}+b \sum_{i=1}^{n} \rho_{i} \sigma_{i}^{2}}{\lambda_{i}^{2}+2 b \sum_{i=1}^{n} \rho_{i} \sigma_{i}^{2}}
$$

The conclusion 1 can be obtained from formula (5) and formula (8).

Conclusion 1: $\beta_{1}^{*}, \beta_{2}^{*}$ increases with the increase of and decreases with the increase of $b$ and $\sum_{i=1}^{n} \rho_{i} \sigma_{i}^{2}$. Comparatively speaking, change magnitude of $\beta^{*}$ with these factors is smaller than that of $\beta_{2}^{*}$ with these factors. it means function of two incentive coefficients on alliance members is $\beta_{1}^{*}<\beta_{2}^{*}$, which means alliance member enterprise are more sensitive to $\beta_{2}^{*}$.

\section{Allocation of Supervision Cost}

It is known from formula (5) and formura (8) that, on premise that $b, \lambda_{i}$ are assumed to be determined, $\beta_{1}^{*}, \beta_{2}^{*}$ mainly depend on risk aversion $\rho_{i}$ of every alliance member enterprise and information varianee $\sigma_{i}^{2}$ observed in output. According to principal agent theory, the larger $\sigma_{i}^{*}$, the smaller incentive obtained by alliance member enterprise. Method to reduce $\sigma_{i}^{2}$ is 10 strengthen supervision, which requires client and alliance leader enterprise to make balance between supervision cost and revenue. What's more, specific supervision cost is difficult to quantized because it increases with the decrease of $\sigma_{i}^{2}$. In group incentive model of agricultural product logistics alliance, supervision cost of alliance leader enterprise is the fixed cost of extra expenditure after one agent is added. Generally, this fixed cost is less than supervision cost calculated by traditional incentive. At this noment, cost will be saved necessarily. The saved cost is actually allocated by allianee member enterprises. If the added enterprise is set as the principal in model (1), it an directly supervise member enterprises. As member enterprises have motivation to raze client enterprises are required to be set as second-level principal to supervise it. Therefore, alliance member enterprises have paid for it and the share of residual value is reduced. Sharing proportion is change from $\beta_{1}$ to $\beta_{1} \beta_{2}$. Because under such allocation system, alliance member enterprise become the claimant of residual value and they ought to assume part of supervision cost.

According to incentive theory, incentive coefficient calculated under the balance between supervision cost and revenue is less than the incentive coefficient in the case without supervision cost. It indicates that alliance member enterprise share less residual value as they actually undertake part of supervision cost. However, cost undertaken by alliance members is recessive and it is difficult for alliance members to sense it. In group 
incentive model, supervision cost of alliance members is explicit. $\beta_{2}$ not only has incentive function but also has restraint function. In group incentive model, supervision cost undertaken by client enterprises is $\left(1-\beta_{1}\right) \alpha$ and total supervision cost undertaken by all the member enterprises is: $\beta_{1}\left(1-\beta_{2}\right)\left[\sum_{i=1}^{n} \lambda_{i} e_{i}-(n+1) \alpha\right]-\beta_{1} \alpha$.

\section{Scale Design of Collaborative Logistics}

Sometimes in agricultural product logistics alliance, multiple logistics enterprises well matched in strength and scale may coexist. They may joint up to compose the "alliance leader decision-making system" of logistics alliance to make decisions about operation of alliance, which also reflects the democracy of the decision to some degree. Assume alliance leader decision-making system exists in logistics alliance, number of enterprises, in alliance leader decision-making system is $m$ and other assumptions are the same as 21 construct the following group incentive model (9).

$$
\begin{aligned}
& \max _{\alpha, \beta_{1}}\left(1-\beta_{1}\right)\left(R-\sum_{i=1}^{n+m} \alpha\right) \\
& \text { s.t. } \max _{\alpha, \beta_{1}, \beta_{2}}\left[m \alpha+\beta_{1}\left(1-\beta_{2}\right)\left(R-\sum_{i=1}^{n+m} \alpha\right)-c\right] \\
& \text { s.t. }(I R) \alpha+\beta_{1} \beta_{2}\left(R\left(e_{i}\right)-\frac{m \alpha}{n}-\alpha\right)-\frac{1}{2} \rho_{i} \beta_{1}^{2} \beta_{2}^{2} \sigma_{i}^{2}-\frac{1}{2} b e_{i}^{2} \geq \bar{\alpha}_{o} \\
& \text { (IC) } \max \left[\alpha+\beta_{1} \beta_{2}\left(R\left(e_{i}^{*}\right)-\frac{m \alpha}{n}-\alpha\right)-\frac{1}{2} \rho_{i} \beta_{1}^{2} \beta_{2}^{2} \sigma_{i}^{2}-b e\right]
\end{aligned}
$$

According to participation constraint in model (9), it can be calculated that:

$\beta_{1}=\frac{b e_{i}}{\beta_{2} \lambda_{i}}, \quad \beta_{2}=\frac{b e_{i}}{\beta_{1} \lambda_{i}}$<smiles>CCCCCCC</smiles>

Derive $e_{i}$ in $\beta_{1}, \beta_{2}$ according to (10) to calculate:

$\frac{\partial \beta_{1}}{\partial e_{i}}=\frac{b}{\beta_{2} \lambda_{i}}, \frac{\partial \beta_{2}}{\partial e_{i}}=\frac{b}{\beta_{1} \lambda_{1}}$

At this moment deterministic equivalent income of logistics alliance is:

$\sum_{i=1}^{n+m} \alpha+\beta_{1}\left(\sum_{i=1}^{n} \lambda_{i} e_{i}-\sum_{i=1}^{n+m} \alpha\right)-\frac{1}{2} \beta_{1}^{2} \beta_{2}^{2} \sum_{i=1}^{n} \beta_{i} \alpha_{i}-\frac{1}{2} b \sum_{i=1}^{n} e_{i}^{2}-m c$

Derive $e_{i}$ in forma (12) by combining (9) and command it to be zero to calculate:

$$
\beta_{1}^{\prime *}=\frac{\lambda_{i}^{2}+b \sum_{i \neq 1}^{n} \rho_{i} \sigma_{i}}{\lambda_{i}^{2}+2 b \sum_{i=1}^{n} \rho_{i} \sigma_{i}^{2}}
$$

Stm of deterministic equivalent incomes of client enterprises and logistics alliance is:

$\sum_{i=1}^{n+1} \lambda_{i} e_{i}-\frac{1}{2}\left(\sum_{i=1}^{n} \rho_{i} \sigma_{i}^{2}\right) \beta_{2} \beta_{1}^{2}-\frac{1}{2} b \sum_{i=1}^{n} e_{i}^{2}-m c$

Derive $e_{i}$ from formula (11) and command it to be 0 by combining (11) to calculate:

$\lambda_{i}-\frac{2 b}{\lambda_{i}}\left(\sum_{i=1}^{n} \rho_{i} \sigma_{i}^{2}\right) \beta_{1} \beta_{2}-\beta_{1} \beta_{2} \lambda_{i}=0$

It can be calculated from formula (15) that:

$\beta_{2}^{\prime *}=\frac{\lambda_{i}^{2}}{\lambda_{i}^{2}+b \sum_{i=1}^{n} \rho_{i} \sigma_{i}^{2}}$ 
Combine formula (5), formula (8), formula (13) and formula (16) to draw conclusion 2.

Conclusion 2: in group incentive model of logistics alliance, the incentive coefficients to set 1 alliance leader enterprise and alliance decision-making system of multiple enterprises are equivalent, which means $\beta_{1}^{*}=\beta_{1}^{\prime *}$ and $\beta_{2}^{*}=\beta_{2}^{\prime *}$.

It can be known that multiple alliance leader enterprises have no impact on $\beta_{1}, \beta_{2}$ .Under circumstances of constant number of alliance members, the increase of alliance leader enterprise will influence the average revenue of alliance enterprises and member enterprises. When the expected utility is less than fixed income, alliance leader enterprise will have no motivation to supervise and members will not have motivation to provide high-level service. Therefore, number of alliance leader enterprises has correlation relationship with alliance group scale.

On the basis of analysis above, assume deterministic equivalent incomes of alliance leader enterprises and member enterprises are equal to their reserved revenue respectively, and output variances, risk aversions and outputs of alliance member enterprises are the same to research the relationship between $m, n$ to draw conclusion 3 .

Conclusion 3: relationship between number of enterprises in alliance leader decisionmaking system and number of alliance members is:

$m=\frac{\left(1-\beta_{1}\right) \alpha+\beta_{1} \lambda_{i} e_{i}-\frac{1}{2} \beta_{1}^{2} \beta_{2}^{2} \rho \sigma^{2}-\frac{1}{2} b e_{i}^{2}-\bar{\alpha}_{0}}{c+\bar{\alpha}_{0}-\left(1-\beta_{1}\right) \alpha} n$

Authentication: assume deterministic equivalent incomes of alliance leader enterprises and member enterprises are equal to their eserved revenue respectively and output variances, risk aversions and outputs of alliance member enterprises are the same and the

$$
\begin{aligned}
& \text { following relation exists. } \\
& \sum_{i=1}^{n+m} \alpha+\beta_{1}\left(\sum_{i=1}^{n} R\left(e_{i}\right)-\sum_{i=1}^{n+m} \alpha\right)-\frac{1}{2} \beta_{2}^{2} \beta_{1}^{2} \rho \sigma^{2}-\frac{1}{2} \sum_{i=1}^{n} b e_{i}^{2}=m c \\
& =(m+n) \bar{\alpha}_{0} \\
& \quad \text { Relationship between } m, n \text { detived from fomula (18) is: }
\end{aligned}
$$

$$
m=\frac{\left(1-\beta_{1}\right) \alpha+\beta_{1} \lambda_{i} e_{i}-\frac{1}{2} \beta_{1}^{2} \beta_{2}^{2} \beta \sigma \frac{1}{2} b e_{i}^{2}-\bar{\alpha}_{0}}{c+\bar{\alpha}_{0}-\left(1-\beta_{1}\right) \alpha}
$$

It is known from formula (19), when $n$ is determined, $m$ increases with increase of $\beta_{1}, \lambda_{i} e_{i}$ and decreases with increases of effort cost of alliance member enterprise. It also explains, when number of enterprises in logistics alliance is relatively large and there is difficulty in supervision number of enterprise in alliance leader decision-making system can be increased approp riately.

Corollary: when $m=1$, minimum group scale $n$ of alliance member enterprise required in setting one alliance enterprise leader enterprise is:

$$
n=\frac{c+\bar{\alpha}_{0}-\left(1-\beta_{1}\right) \alpha}{(1-\beta) \alpha \beta_{1} \lambda_{i} e_{i}-\frac{1}{2} \beta_{1}^{2} \beta_{2}^{2} \rho \sigma^{2}-\frac{1}{2} b e_{i}^{2}-\bar{\alpha}_{0}}
$$

Formula (20) explains, in operation process of logistics alliance, only when then number of alliance number achieves a certain scale, it is significant to increase the number of enterprises in alliance leader decision-making system.

\section{Conclusion}

As "hitchhike" is common in logistics alliance, it is an important task for alliance leader enterprises and alliance leader decision-making system in the alliance to resolve hitchhike problem. One of the main reasons for common "hitchhike" in logistic alliance is the output of alliance is common. Individual contribution of every alliance member is hard to distinguish, which results in the motivation to laze for some alliance members. In consideration of the existing problem above, the thesis draws the following conclusions 
based on double-layer principal agent theory: comparing incentive coefficients $\beta_{1}^{*}, \beta_{2}^{*}$ of client enterprises and alliance enterprises, incentive coefficient $\beta_{2}^{*}$ alliance member enterprises set for alliance leader enterprises is more sensitive; in the group incentive model of collaborative logistics alliance, incentive coefficients to set 1 alliance leader enterprise and alliance leader decision-making system including multiple enterprise are equivalent; when the number of member enterprises in collaborative logistics alliance is relatively large and there is difficulty in supervision, number of enterprise in alliance leader decision-making system can be increased appropriately and scale of logistics alliance can be determined according to relationship between number of enterprises in alliance leader decision-making system and number of alliance member enterprise; in operation process of logistics alliance, only when then number of alliance number achieves a certain scale (when $m=1$ ), it is significant to increase the number of enterprises in alliance leader decision-making system.

\section{Acknowledgement}

The research is supported by The National Natural Science Foundation of China "link mode, evolutionary mechanism an its optimization management of plank yariety rights value chain" under Grant No.71173138; The Natural Science Foundation of Shandong Province "Research on the innovative service platiorm of mould based on the high-end industrial concentration area of Jiaodong Peninsula" under Grant No. ZR2010GL008 and The National Social Science Foundation of China "The endowment effect and path selection of affirming the rights of the homestead and population urbanization" under Grant No. 15BJL055.

\section{References}

[1] Z. Lv, T. Yin, Y. Han. WebVR virtual Teality engine based on P2P network[J]. Journal of Networks, (2011), vol. 6, no. 7,pp. 990-998.

[2] J. Yang, J. Zhou, Z. Lv, “A Real-Time Monitorng System of Industry Carbon Monoxide Based on Wireless Sensor Networks[J]", Sensors, (2015), vol. 15, no. 11, pp. 29535-29546.

[3] D. Jiang, X. Ying, Y. Han, "Collaborative multi-hop routing in cognitive wireless networks[J]", Wireless Personal Communications, (2016), 86(2): 901-923.

[4] Z. Lv, A. Halawan, S. Feng, "Multimodal hand and foot gesture interaction for handheld devices[J]", ACM Transactions on Multimedia, Computing, Communications, and Applications (TOMM), (2014), $11(1 \mathrm{~s})$.

[5] J. Hu and 7. Gao, "Distinction immune genes of hepatitis-induced heptatocellular carcinoma[J]", Bioinformatics, (2012), vol. 28, no. 24, pp. 3191-3194.

[6] J. Hu, Z. Gao and W. Pan, "Multiangle Social Network Recommendation Algorithms and Similarity Network Evaluation[J]", Journal of Applied Mathematics, 2013 (2013).

[7] J. Hu and Z. Gao, "Modules identification in gene positive networks of hepatocellular carcinoma using Pearson agglomerative method and Pearson cohesion coupling modularity[J]", Journal of Applied Mathematics, (2012).

[8] Y. Geng, J. Chen, Fu, R., Bao, G., and K. Pahlavan, "Enlighten Wearable Physiological Monitoring systems. On-Body RF Characteristics Based Human Motion Classification Using a Support Vector Machine", pp. (99), pp. 1-16.

9] X. Song, and Y. Geng, "Distributed Community Detection Optimization Algorithm for Complex Networks", Journal of Networks, 9(10), 2758-2765.

[10] Pahlavan, K., Krishnamurthy, P., and Geng, Y. Localization Challenges for the Emergence of the Smart World, Access, IEEE, vol. 3, no. 1, pp. 1-11.

[11] J. He, Y. Geng, Y. Wan, S. Li, and K. Pahlavan, (2013). A cyber physical test-bed for virtualization of RF access environment for body sensor network. Sensors Journal, IEEE, vol. 13, no. 10, pp. 3826-3836

[12] D. Jiang, Z. Xu , Z. Lv, “ A multicast delivery approach with minimum energy consumption for wireless multi-hop networks[J]", Telecommunication Systems, (2015), pp. 1-12.

[13] Y. Lin, J. Yang, Z. Lv, "A self-assessment stereo capture model applicable to the internet of things[J]", Sensors, (2015), vol. 15, no. 8, pp. 20925-20944.

[14] Y. Liang*. "Satisfaction With Economic and Social Rights and Quality of Life in a Post-Disaster Zone in China: Evidence From Earthquake-Prone Sichuan", Disaster Medicine and Public Health Preparedness, vol. 9, no. 2, pp. 111-118. 
[15] Y. Liang*, "Correlations Between Health-Related Quality of Life and Interpersonal Trust: Comparisons Between Two.

[16] Generations of Chinese Rural-to-Urban Migrants, Social Indicators Research, vol. 123, no. 3, pp. $\quad 677-700$.

[17] Y. Liang*, Peiyi Lu.(2014). Medical insurance policy organized by Chinese government and the health inequity of the elderly: longitudinal comparison based on effect of New Cooperative Medical Scheme on health of rural elderly in 22 provinces and cities, International Journal for Equity in Health, 13:37, 115. DOI:10.1186/1475-9276-13-37.

[18] Y. Liang*, D. Zhu,Subjective Well-Being of Chinese Landless Peasants in Relatively Developed Regions: Measurement Using PANAS and SWLS. Social Indicators Research, vol. 123, no. 3, pp. 817-835.

[19] Y. Liang*, X. Wang, "Developing a new perspective to study the health of survivors of Sichuan earthquakes in China: a study on the effect of post-earthquake rescue policies on survivors' healthrelated quality of life, Health Research Policy and Systems, 11:41,1-12. DOI:10.1186/1478-4505-11-41

[20] J. Hu and Z. Gao, "Modules identification in gene positive networks of hepatocellular carcinoma using Pearson agglomerative method and Pearson cohesion coupling modularity[J]", Journal of Applied Mathematics, 2012 (2012).

[21] Y. Geng, J. Chen, R. Fu, G. Bao, and K. Pahlavan, (2015), "Enlighten Wearable Physiological Monitoring systems", On-Body RF Characteristics Based Human Motion Classification Using a Support Vector Machine. PP(99), pp. 1-16.

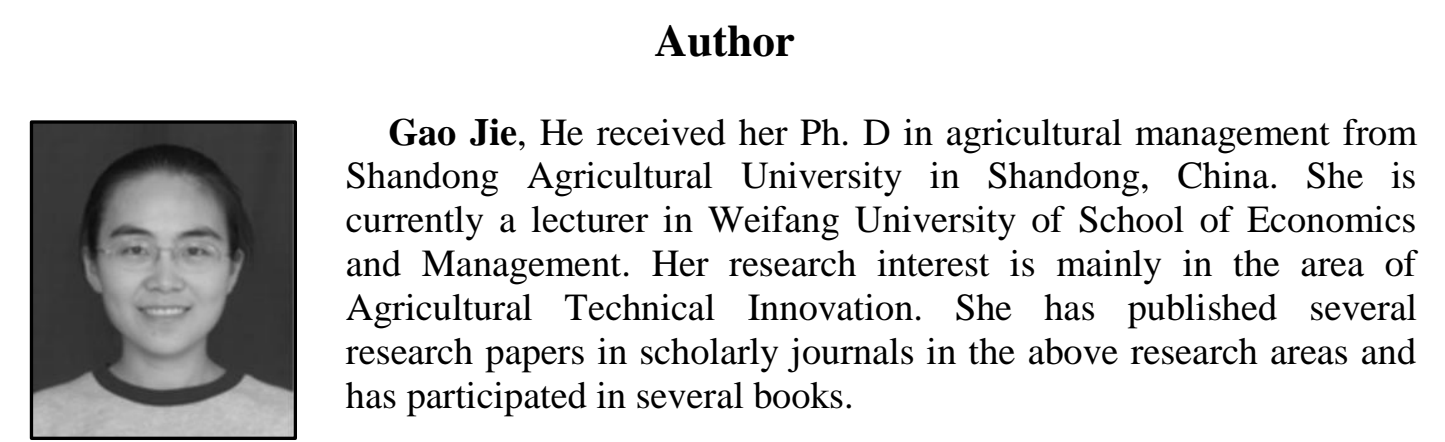
has participated in several books.

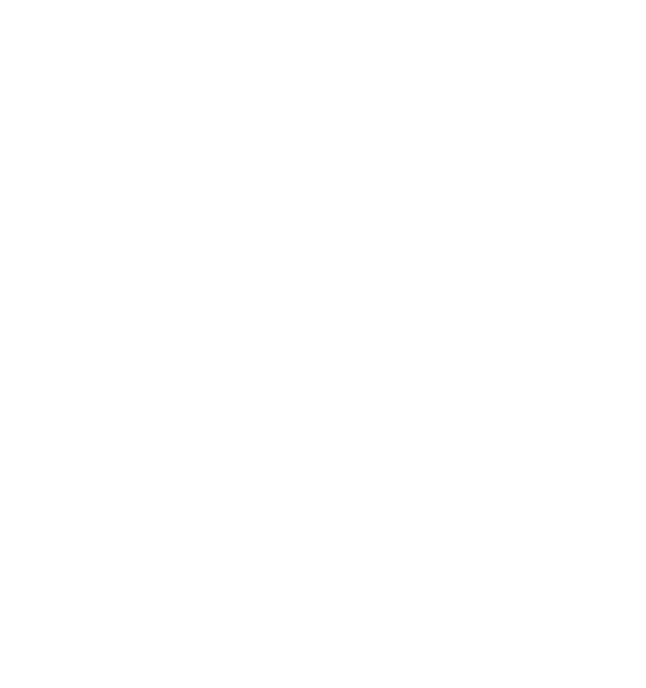

